\begin{tabular}{|c|c|c|}
\hline & Journal Homepage: - www.journalijar.com & $\begin{array}{l}\text { INTERNATIONAL JOURNAL OF } \\
\text { ADVANCED RESEARCH (JIAR) }\end{array}$ \\
\hline D. 2320-5407 & $\begin{array}{l}\text { Article DOI: } 10.21474 / \mathrm{IJAR} 01 / 7840 \\
\text { DOI URL: http://dx.doi.org/10.21474/IJAR01/7840 }\end{array}$ & \\
\hline
\end{tabular}

RESEARCH ARTICLE

\title{
PANCREATIC METASTASIS OF RENAL ADENOCARCINOMA.
}

Malika Amarir, Omar Jellouli and Laila Jroundi.

Emergency radiology Department, UH avicenne ,university Mohammed V ,Rabat .Morocco.

\section{Manuscript Info}

\section{Manuscript History}

Received: 8 August 2018

Final Accepted: 10 September 2018

Published: October 2018

\section{Keywords:}

Pancreatic metastasis; Kidney cancer; radiological monitoring

\begin{abstract}
Pancreatic metastasis of kidney cancer is very rare, accounting for less than 0.25 to $3 \%$ of cases. The detection of metastasis is often fortuitous in surveillance after renal cancer resection. We report a case of pancreatic metastases of renal adenocarcinoma. This observation emphasizes the advantage of the radiological assessment for early diagnosis, which allows to discuss a curative surgical act which seems to be the only treatment able to prolong the survival of the patients
\end{abstract}

Copy Right, IJAR, 2018,. All rights reserved.

\section{Introduction:-}

Les localisations secondaires des cancers du rein sont le plus souvent de siège pulmonaire, hépatique ou osseux. Les métastases pancréatiques sont exceptionnelles et ne représentent que 2 à $4 \%$ des lésions malignes pancréatiques. Nous rapportons une nouvelle observation de deux métastases pancréatiques tardive d'un adénocarcinome à cellules claires rénales.

\section{Observation:-}

Il s'agit d'une femme de 60 ans opérée 10 ans auparavant pour une néphrectomie droite élargie pour adénocarcinome rénal à cellules clairs, ayant été perdue de vue, qui consulte pour douleur abdominale diffuse, l'interrogatoire révèle des douleurs périodiques,l'examen clinique est sans particularité en dehors de la cicatrice de la néphrectomie, une TDM abdominale sans et avec injection de PCI est réalisée ayant objectivé la présence au niveau du corps et de la queue du pancréas de deux nodules tissulaires arrondies hypervasculaires après injection figure 1,2 


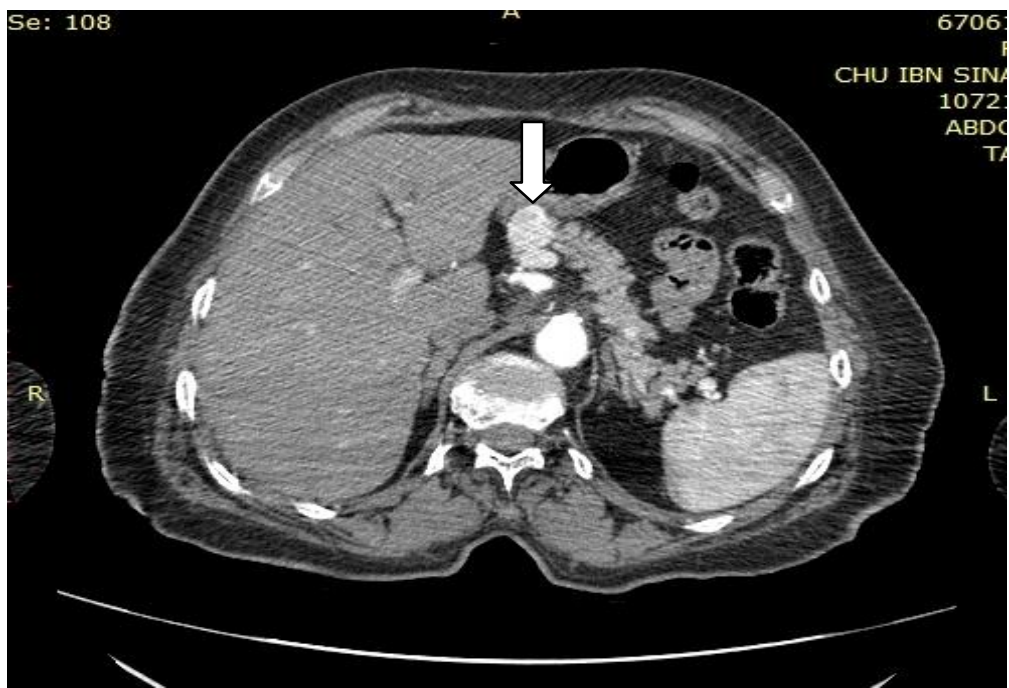

Figure 1:-TDM abdominale coupe axiale avec injection de PCI : lésion au niveau du corps du pancréas (flèche) rehaussée après injection de PCI

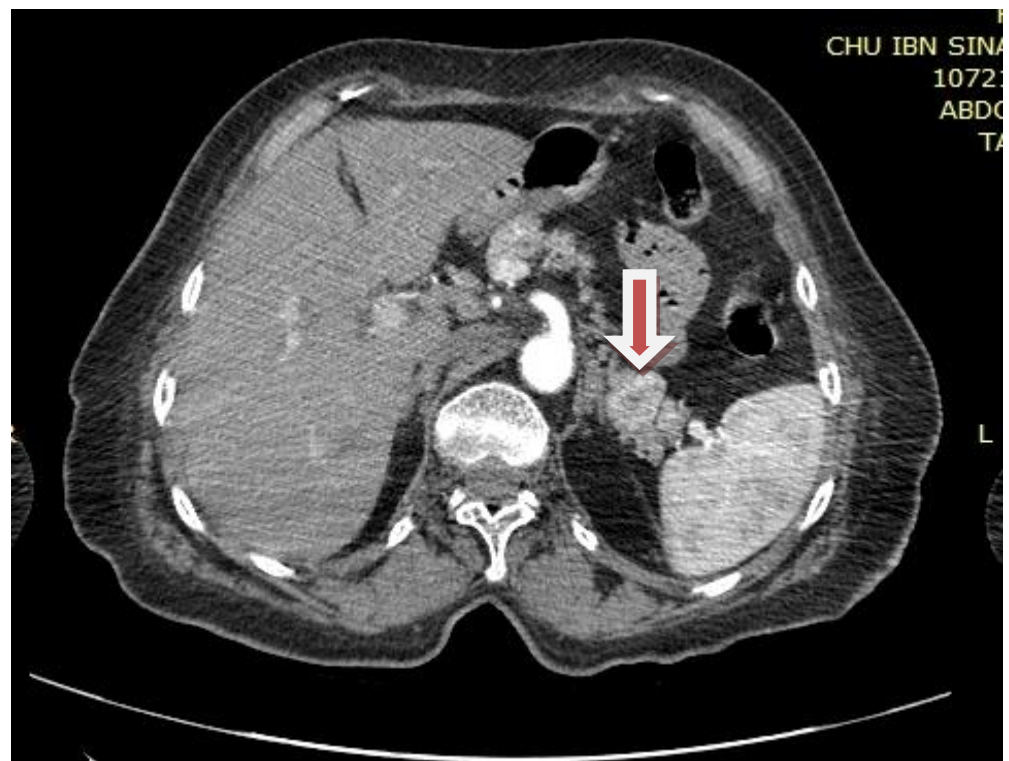

Figure 2:-TDM abdominale coupe axiale avec injection : présence d'une lésion grossièrement arrondie au niveau de la queue du pancréas (flèche) rehaussée après injection du PCI

\section{Discussion:-}

Les métastases des cancers du rein au niveau du pancréas sont peu fréquentes et se voient de façon tardive ; représentant 0,25 à $3 \%$ [1.2.3]. Le sex ratio est de 1,7 en faveur du sexe masculin, comparable à celui de l'épidémiologie du cancer du rein.Lors du diagnostic, l'âge moyen est de 61 ans avec des extrêmes allant de 39 à 76 ans dans la littérature [4]. Le délai moyen séparant la néphrectomie et la survenue des métastases est long avec une moyenne de 10 ans (comme dans notre cas) mais elles peuvent apparaître plus précocement voire conjointement à la tumeur rénale [5]. La détection des métastases survient de façon fortuite lors des examens de surveillance après la résection du cancer rénal [7]. La symptomatologie des métastases pancréatiques est peu spécifiques [5]. Elles peuvent se manifester par des algies abdominales, une pancréatite aiguë, une insuffisance pancréatique exo ou endocrine ,une altération de l'état général, un ictère par compression du cholédoque ou une hémorragie digestive par l'envahissement pariétale du duodénum sont les symptômes les plus fréquemment rapportés [6]. Ces métastases sont le plus souvent de localisation corporéo-caudale [8]. Elles sont le plus souvent uniques $85 \%$ des cas [9].contrairement à une double localisation chez notre patiente.Les métastases pancréatiques des cancers du rein peuvent poser des difficultés de diagnostic radiologique. la glande pancréatique est mal explorée par l'échographie 
abdominale qui n'a aucune spécificité pour le diagnostic des lésions secondaires [10] une hétérogénéité de la glande peut être décrite [11]. L'écho-doppler a une meilleure sensibilité puisqu'il montre le profil hyper vasculaire de la lésion [12]. Le scanner abdominale révèle une lésion hypervasculaire, parfois hétérogène, aux dépens du pancréas. le caractère hyper vascularisé de ces métastases se manifeste par une lésion qui se rehausse après injection du produit de contraste, contrairement a l'hypovascularisation des tumeurs primitives du pancréas $[1,2,13,14]$..L'IRM donne les mêmes informations que la TDM mais avec une meilleur sensibilité de la lésion avant l'injection de contraste du fait du signal nettement diffèrent de celui du parenchyme pancréatique hypointense en T1 et hyper intense en T2 avec un caractère hyper vasculaire au temps artériel ou hétérogène en fonction de sa taille [21]. L'artériographie coeliomésentérique n'est plus réalisée de nos jours en raison de la qualité des examens scannographiques actuellement disponibles. En écho-endoscopie, la métastase apparaît sous forme dune masse solide homogène avec souvent une plage centrale hypoéchogène traduisant la nécrose. Cet examen est difficile à interpréter lorsque la métastase est volumineuse ou occupe la queue du pancréas [11]. le diagnostic de certitude reste histologique après ponction biopsie de la lésion ou résection chirurgicale cependant cet examen n'est pas réalisable en raison du caractère éventuellement hémorragique de ces métastases [7,11]. Néanmoins, la ponction n'est justifie que si aucune tumeur primitive n'est retrouvée, si l'aspect de la lésion pancréatique oriente vers un autre type histologique (en cas de masse pancréatique hypervasculaire, une tumeur endocrine représente le principal diagnostic différentiel), si l'exérèse de la lésion n'est pas faisable $[7,11,15]$. ou si le délai entre le diagnostic de la lésion pancréatique et la néphrectomie est important et rend le diagnostic de métastase moins plausible [16,17].Le traitement des métastases pancréatiques est chirurgical [18]. L'exérèse pancréatique est le plus souvent élargie en fonction du siège de la lésion [7, 19]. Une duodénopancréatectomie céphalique ou une splénopancréatectomie sont le plus souvent inévitables [15]. La chimiothérapie par vinblastine ou l'immunothérapie par interféron alpha n'ont pas montré leur efficacité dans le traitement de ces métastases, mais elles peuvent être utiles après une chirurgie d'exérèse avec localisation ganglionnaire ou residu tumoral minime $[11,20]$.La survie après résection chirurgicale des métastases pancréatiques est satisfaisante avec une médiane de survie de 30 mois. Les facteurs de bon pronostic de ces métastases pancréatiques sont; la survenue tardive, une forme asymptomatique, une lésion pancréatique unique et/ou a centre nécrotique [11].

\section{Conclusion:-}

Les localisations pancréatiques secondaires des cancers du rein sont de survenue rarissime et souvent tardive. Elles sont décelées habituellement au cours du bilan de surveillance du cancer du rein. Le diagnostic précoce permet de discuter un acte chirurgical curatif qui parait être le seul traitement capable de prolonger la survie des patients.

\section{Pas de conflits d'intérêts.}

\section{Bibliographie:-}

1. Machado NO, Chopra P. Pancreatic Metastasis from renal carcinoma managed by whipple resection pattern, Surgical Management and Outcome. A Case Report and Literature Review of Metastatic JOP. J Pancreas. 2009;10:413-8. [PubMed]

2. Ninan S, Jain PK, Paul A, et al. Synchronous pancreatic metastases from asymptomatic renal cell carcinoma. JOP. J Pancreas (Online) 2005;6:26-8. [PubMed]

3. Zerbi A, Ortolano E, Balzano G, et al. Pancreatic metastasis from renal cell carcinoma: which patients benefit from surgical resections? Ann SurgOncol. 2008;15:1161-

4. MASSON J., BEREDER I., RAGNI E., MASSON J.C., RICHAUD C. : Cancer du rein et métastases pancréatiques. A propos de 3 cas. Prog. Urol., 1996; $6: 443-446$.

5. KASSABIAN A., STEIN J., JABBOUR N., PARSA K., SKINNER D., PAREKH D., COSENZA C., SELBY R. : Renal cell carcinoma metastatic to the pancreas : asingle-institution series and review of the literature. Urology, $2000 ; 56: 211-215$.

6. Thompson L, Heffess C. Renal cell carcinoma to the pancreas in surgical pathology material. Cancer 2000;89:1076-88.

7. PESCHAUD F., CHEYNEL N., HAGRY O., TREMEAUX J.C., RAT P., FAVRE J.P. : Surgical treatment of pancreatic metastases from renal carcinoma. Ann.Chir., $2002 ; 127: 527-531$.

8. Stankard CE, Karl RC. The treatment of isolated pancreatic metas- tases from renal cell carcinoma : a surgical review. Am J Gastroen- terol 1992;87:1658-60. 
9. Portal I, Barthet M, Alemy M, Payan MJ, Sastre B, Sahel J. Métastases pancréatiques récidivantes d'un hypernéphrome. Presse Med 1992;21:1822.

10. GHAVAMIAN R., KLEIN K.A., STEPHENS D.H., WELCH T.J., LEROY A.J., RICHARDSON R.L., BURCH P.A., ZINCKE H. : Renal cell carcinoma metastatic to the pancreas: clinical and radiologic features. Mayo Clin. Proc., $2000 ; 75$ : 581-585.

11. F. Peschaud, N. Cheynel, O. Hagry, J.C. Tremeaux, P. Rat, J.P. Favre Traitement chirurgical des métastases pancréatiques des cancers du rein Surgicaltreatment of pancreaticmetastasesfromrenalcarcinoma Reçu le 28 janvier 2002 ; accepté le 24 juin 2002

12. FLATH B., RICKES S., SCHWEIGERT M., LOCHS H., POSSINGER K., WERMKE W. :Differentiation of a pancreatic metastasis of a renal cell carcinoma from aprimary pancreatic carcinoma by echo-enhanced power Doppler sonography. Pancreatology, $2003 ; 3: 349-351$.

13. Faure J-P, Richer J-P, Irani J, et al. Cancer du rein et métastases pancréatiques tardives. À propos de 3 cas et revue de la littérature. ProgUrol. 1998;8:404-7. [PubMed]

14. Reddy S, Edil BH, Cameron JL, et al. Pancreatic resection of isolated metastases from nonpancreatic primary cancers. Ann Surg Oncol. 2008;15:3199-206. http://dx.doi.org/10.1245/s10434-008-0140-7.[PubMed]

15. ROBBINS R.G., FRANCESCHI D., BARKIN J.S. : Solitary metastatic tumors to the pancreas : a case report and review of the literature. Am. J. Gastroenterol.,1996; 91 : 2414-2417.

16. Butturini G, Bassi C, Falconi M, Salvia R, Caldiron E, Iannucci G. Surgical treatment of pancreatic metastases from renal cell carcino- mas. Dig Surg 1998;15:241-6.

17. Klein KA, Stephens DH, Welch TJ. CT characteristics of metastatic disease of the pancreas. Radiographics 1998;18:369-78.

18. RIVOIRE M., VOIGLIO E.J. : Late pancreatic metastases from renal cell carcinoma. Surgery, $1996 ; 119: 240$.

19. HIROTA T., TOMIDA T., IWASA M., TAKAHASHI K., KANEDA M., TAMAKI H. : Solitary pancreatic metastasis occurring eight years after nephrectomy for renalcell carcinoma. A case report and surgical review. Int. J. Pancreatol., 1996 ; $19: 145-153$.

20. SOHN T.A., YEA C.J., CAMERON J.L., NAKEEB A., LILLEMOE K.D. : Renal cell carcinoma metastatic to the pancreas : results of surgical management. J.Gastrointest. Surg., $2001 ; 5: 346-351$.

21. ROY C.CHAUDRON V. et al metastasepancreatiquesmetachrones des carcinomes rénaux : role de l'imagerie à propose de 17 patiente avec correlations chirurgicales journal de radiologie (2011) 92,1091-1100 